\title{
A retrospective analysis of cardiovascular adverse events associated with immune checkpoint inhibitors
}

\author{
Jessica Castrillon Lal ${ }^{1,2}$, Sherry-Ann Brown ${ }^{3}$, Patrick Collier ${ }^{2,4}$ and Feixiong Cheng ${ }^{1,2,5^{*}}$
}

\begin{abstract}
Background: Modern therapies in oncology have increased cancer survivorship, as well as the incidence of cardiovascular adverse events. While immune checkpoint inhibitors have shown significant clinical impact in several cancer types, the incidence of immune-related cardiovascular (CV) adverse events poses an additional health concern and has been reported.

Methods: We performed a retrospective analysis of the FDA Adverse Event Reporting System data of suspect product reports for immunotherapy and classical chemotherapy from January 2010-March 2020. We identified 90, 740 total adverse event reports related to immune checkpoint inhibitors and classical chemotherapy.

Results: We found that myocarditis was significantly associated with patients receiving anti-program cell death protein 1 (PD-1) or anti-program death ligand 1 (PD-L1), odds ratio (OR) $=23.86$ (95\% confidence interval [CI] 11.7648.42, (adjusted $p$-value) $q<0.001$ ), and combination immunotherapy, $\mathrm{OR}=7.29$ (95\% Cl 1.03-51.89, $q=0.047$ ). Heart failure was significantly associated in chemotherapy compared to PD-(L)1, OR $=0.50$ ( $95 \% \mathrm{Cl} 0.37-0.69$, $q<0.001), \mathrm{CTLA} 4, \mathrm{OR}=0.08$ (95\% Cl 0.03-0.20, $q<0.001$ ), and combination immunotherapy, OR= 0.25 (95\% Cl $0.13-0.48, q<0.001$ ). Additionally, we observe a sex-specificity towards males in cardiac adverse reports for arrhythmias, $\mathrm{OR}=0.81(95 \% \mathrm{Cl} 0.75-0.87, q<0.001)$, coronary artery disease, $0.63(95 \% \mathrm{Cl} 0.53-0.76, q<0.001)$, myocardial infarction, $\mathrm{OR}=0.60(95 \% \mathrm{Cl} 0.53-0.67, q<0.001)$, myocarditis, $\mathrm{OR}=0.59(95 \% \mathrm{Cl} 0.47-0.75, q<0.001)$ and pericarditis, $\mathrm{OR}=0.5$ (95\% Cl $0.35-0.73, q<0.001)$.

Conclusion: Our study provides the current risk estimates of cardiac adverse events in patients treated with immunotherapy compared to conventional chemotherapy. Understanding the clinical risk factors that predispose immunotherapy-treated cancer patients to often fatal CV adverse events will be crucial in Cardio-Oncology management.
\end{abstract}

Keywords: Cardio-oncology, Cardiotoxicity, Immune checkpoint inhibitors, Immunotherapy, Myocardial infarction, Myocarditis

\footnotetext{
* Correspondence: chengf@ccf.org

'Genomic Medicine Institute, Lerner Research Institute, Cleveland Clinic, Cleveland, OH 44195, USA

${ }^{2}$ Department of Molecular Medicine, Cleveland Clinic Lerner College of Medicine, Case Western Reserve University, Cleveland, OH 44195, USA

Full list of author information is available at the end of the article
}

(C) The Author(s). 2021 Open Access This article is licensed under a Creative Commons Attribution 4.0 International License, which permits use, sharing, adaptation, distribution and reproduction in any medium or format, as long as you give appropriate credit to the original author(s) and the source, provide a link to the Creative Commons licence, and indicate if changes were made. The images or other third party material in this article are included in the article's Creative Commons licence, unless indicated otherwise in a credit line to the material. If material is not included in the article's Creative Commons licence and your intended use is not permitted by statutory regulation or exceeds the permitted use, you will need to obtain permission directly from the copyright holder. To view a copy of this licence, visit http://creativecommons.org/licenses/by/4.0/. The Creative Commons Public Domain Dedication waiver (http://creativecommons.org/publicdomain/zero/1.0/) applies to the data made available in this article, unless otherwise stated in a credit line to the data. 


\section{Introduction}

Immune checkpoint inhibitors (ICIs) have increased cancer survivorship and are now standard of care for numerous cancer types [1-3]. Antibodies targeting programmed cell death protein (PD-1), programmed deathligand (PD-L1), and cytotoxic T-lymphocyte-associated protein 4 (CTLA4) work to re-invigorate the immune system to recognize and lyse tumors. ICIs have now gained 67 FDA approvals in over 17 cancer types, and two tissue agnostic conditions $[4,5]$. However, adverse events (AE) are common in patients receiving antiCTLA4 (89\%) and anti-PD-1 or PD-L1 (PD-(L)1) (74\%) therapies. Virtually all patients receiving more than one ICI experience adverse events (90\%) [6]. While cardiac adverse events comprise less than $1 \%$ of all $\mathrm{AE}$, they are disproportionally more lethal [6]. It is unclear whether this low incidence results from a lack of reporting or misdiagnosis in part due to heterogeneity in clinical presentation. Cardiac AE encompasses a diverse set of disorders in the heart including myocarditis, pericarditis, arterial vascular disease, venous thromboembolism, pulmonary hypertension, arrhythmias, and heart failure. Further complicating the differential diagnosis and reporting, is the fact that more than one immune-related adverse event may occur with another [7]. Which clinical features predispose cancer patients to immune-associated cardiac AE remains poorly understood. As increased numbers of new targeted and immune-based therapies enter the market, the management of cancer patients continues to become more complicated with an increased need for predictive biomarkers $[8,9]$.

Pharmacovigilance studies prior to 2018 have raised awareness of the incidence of cardiac AE following ICI, leading to more case reports in the past 2 years (Additional File 1: Supplemental Table 1) [8, 10, 11]. Therefore, we sought to analyze and characterize cardiac adverse events associated with ICI in recent years. To achieve this, we mined case reports from the FDA Adverse Event Reporting System to determine if ICIassociated cardiac adverse events are disproportionally more frequent compared to classical chemotherapy based on available information on all adverse events related to the selected therapies.

\section{Methods}

\section{FAERS adverse events data extraction}

The FDA Adverse Event Reporting System obtains reports submitted to the FDA by the drug manufacturers, health care providers, and consumers [12]. All adverse events case reports pertaining to anti-PD1, anti-PD-L1, anti-CTLA4, and classical chemotherapy agents (listed in Additional File 1: Supplemental Table 2) and were extracted from Jan 2011-March 2020 in July 2020, using drug name keywords. Adverse events are entered using terms in the Medical Dictionary for Regulatory Activities (MedDRA) terminology (listed in Additional file 3) (http://www.meddramsso.com/index.asp). Case reports include information on causal medication, the reason for use, other medications currently in use, reaction, and case demographics.

\section{Data Processing \& Statistical Analysis}

We performed categorical classification for cases who received cardioprotective medications and/or oral steroids by text mining of medication keywords in the "Concomitant Product Names" column of the FAERS data using terms listed in Additional File 1: Supplemental Table 3. Cancer histology classification was performed according to the international classification of diseases (ICD9/10) for oncology and nomenclature of histologies (see Additional file 2). To overcome discrepancies of numerous terms or phrases used for the same cardiac adverse events, keywords for reactions were inspected and categorized to cardiac adverse event groups- myocarditis, pericarditis, myocardial infarction, coronary artery disease, arrhythmia, and heart failure (terms listed in Additional file 3). Text mining was performed using stringr $\mathrm{R}$ package (v1.4.0) [13]. The data pre-processing includes removal of outliers and missing variables for age, weight, and sex, cases of age $<$ 18, as well as duplicates (Additional File 1: Supplemental Figure 1). The final study cohort included 90,740 cases. We performed a logistic regression analysis of matched cases (1:1) for sex, age, and weight. Case matching was performed using the MatchIt (v3.0.2) R package, and methods were set to "nearest" (nearest neighbor matching) [14]. Odds ratio and their corresponding 95\% confidence intervals were calculated using the epiR (v1.0-15) and epiDisplay (v3.5.0.1) packages $[15,16]$. Likelihood ratio test was used to calculate significance. Adjusted $p$-values with the threshold for statistical significance was set to $q$ (adjusted p-value) $<0.05$. The model was adjusted for age, weight, sex, oral steroid use, cardioprotective medication use, and chemotherapy use. Forest plots were created to display statistical summaries using the metafor $\mathrm{R}$ package (v2.4-0) [17]. All statistical analysis was performed in R (version 4.0.3).

\section{Results \\ Elevated cardiovascular risk of immune checkpoint inhibitors}

To evaluate the frequency of cardiac adverse events in patients treated with ICIs compared to classical chemotherapy, we performed a retrospective analysis using the FDA Adverse Event Reporting System. All adverse events case reports for anti-PD1, anti-PD-L1, anti-CTLA4 therapies, and classical chemotherapy agents were extracted in July 2020 (Additional File 1: Supplemental Table 2). Cohort 
demographics are displayed in Table 1. Following data processing and confounding factor adjustment (see Data Processing \& Statistical Analysis Methods), the number of all adverse case reports was 90,740. For continuous variables, the mean and the interquartile range are displayed. Cardiovascular adverse events accounted for $9.1 \%$ of all adverse event $(n=8300)$. Anti-PD-(L)1, anti-CTLA4, and more than one ICI accounted for 20.4, 2.0, and 4.9\% respectively; chemotherapy accounted for $72.6 \%$ of cases. We used logistic regression models to estimate the odds ratios (OR) and their $95 \%$ confidence intervals $(\mathrm{CI})$ of matched cases, and found myocarditis and heart failure significantly correlated with ICIs and classical chemotherapy, respectively.

Using chemotherapy as a reference group, we found myocarditis was significantly higher in patients receiving combination immunotherapy, odds ratio $(\mathrm{OR})=7.29$ (95\% confidence interval $[\mathrm{CI}] 1.03-51.89, q=0.047)$, anti-program cell death protein 1 (PD-1), or antiprogram death-ligand 1 (PD-L1), OR = 23.86 (95\% confidence interval $[\mathrm{CI}] 11.76-48.42, q$ (adjusted $p$-value) < 0.001) We also found that combination immunotherapy presented a higher risk of myocarditis compared to antiPD-(L)1, OR $=1.53$ (95\% CI 1.02-2.29, $q=0.038)$, and anti-CTLA4, OR $=4.97$ (95\% CI 2.03-12.20, $q<0.001$ ),

Table 1 FDA Adverse Event Reporting System analysis case demographics

\begin{tabular}{ll}
\hline Characteristics & $\mathbf{n}(\%)$ \\
\hline Total & 90,740 \\
Age & $63(53-70)$ \\
$\quad$ Male & \\
Female & $41,162(45.4 \%)$ \\
Weight (kg) & $49,578(54.6 \%)$ \\
Cardiac Adverse Events & $73.5(59.8-84.0)$ \\
Myocarditis & $8300(9.1 \%)$ \\
Pericarditis & $345(0.4 \%)$ \\
Heart Failure & $143(0.2 \%)$ \\
Myocardial Infarction & $1706(1.9 \%)$ \\
Arrhythmias & $1594(1.8 \%)$ \\
Coronary Artery Disease & $3858(4.3 \%)$ \\
Anti-inflammatory medication use & $654(0.7 \%)$ \\
Cardioprotective medication use & $18,797(20.7 \%)$ \\
Treatment groups & $23,372(25.8 \%)$ \\
anti-PD-(L)1 & \\
anti-CTLA4 & $18,536(20.4 \%)$ \\
combination immunotherapy & $1855(2 \%)$ \\
Chemotherapy & $4442(4.9 \%)$ \\
\hline PD-(L)1 = Programmed cell death protein -1 and Programmed death ligand \\
-1 therapies; CTLA cytotoxic T-lymphocyte-associated protein 4
\end{tabular}

alone (Fig. 1a). This dataset included the most myocarditis cases (345) in respect to previous reports using the FAERS data. We did not observe a difference in the incidental risk of myocarditis between anti-PD-1 and anti-PD-L1 treatments, (Additional File 1: Supplemental Figure 2). We next evaluated the frequency of myocarditis among tumor histologies and found that non-small cell lung cancer (61 cases), melanoma (60 cases), and renal cell carcinoma (57 cases) had the most reports with all cases attributed to ICIs (Fig. 1b). Overall, we find that myocarditis is disproportionally more common following immunotherapy (Fig. 1, Additional File 1: Supplemental Table 1).

\section{Sex-specific cardiovascular risk of immune checkpoint inhibitors}

Using chemotherapy as a reference group, we found that the risk of heart failure was significantly lower in patients receiving anti-CTLA4, OR $=0.08(95 \% \mathrm{CI}$ $0.03-0.20, q<0.001)$, anti-PD-(L)1, OR $=0.50 \quad(95 \%$ CI $0.37-0.69, q<0.001$ ), and combination immunotherapy, OR $=0.25$ (95\% CI 0.13-0.48, $q<0.001$ ) (Fig. 2a). Breast cancer represented the largest case reports for heart failure (373 cases), the majority of which were attributed to classical chemotherapy. We also report sex-specificity in females experiencing heart failure following chemotherapy, $\mathrm{OR}=1.16(95 \%$ CI 1.04-1.29, $q=0.006)$ (Fig. $2 \mathrm{~b}$, Fig. 3).

Recent interest has shed light on sex differences in numerous clinical phenotypes. We were interested to see whether sex differences extend to cardiac adverse events. Indeed, the following cardiac adverse events were more commonly reported in male individuals (odds ratio less than 1): myocarditis $(\mathrm{OR}=0.59,95 \% \mathrm{CI} 0.47-0.75$, $q<0.001$ ), pericarditis (OR $=0.50,95 \%$ CI $0.35-0.73$, $q<0.001)$, myocardial infarction ( $\mathrm{OR}=0.60,95 \% \mathrm{CI}$ $0.53-0.67, \mathrm{q}<0.001)$, coronary artery disease $(\mathrm{OR}=0.63$, $95 \%$ CI $0.53-0.76, q<0.001)$, and arrhythmias $(\mathrm{OR}=0.81$, 95\% CI 0.75-0.87, $q<0.001$ ) (Fig. 3).

We also performed a logistic regression analysis of unmatched cases. We observed that chemotherapy had a higher risk for arrhythmias compared to combination immunotherapy, OR $=0.71$ (95\% CI 0.61-0.84, $q<0.001$ ), and anti-PD-(L)1, OR $=0.67 \quad(95 \% \quad \mathrm{CI} \quad 0.61-0.73$, $q<0.001$ ) therapy (Table 2). However, when testing the effect of individual confounders on model performance, we do not see that the use of cardioprotective medications correlated with arrhythmia adverse events $(\mathrm{OR}=1.0,95 \%$ CI $0.93-1.1, q=0.955)$. We also show chemotherapy has a higher risk for myocardial infarction compared to PD-(L)1 monotherapy $(\mathrm{OR}=0.44,95 \%$ CI $0.34-0.58, q<0.001)$, CTLA-4 monotherapy $(\mathrm{OR}=0.56,95 \%$ CI $0.36-0.85, q=$ $0.007)$, and combination immunotherapy (OR $=0.42,95 \%$ CI $0.29-0.6, q<0.001)$. Overall, our analysis reveals sex 


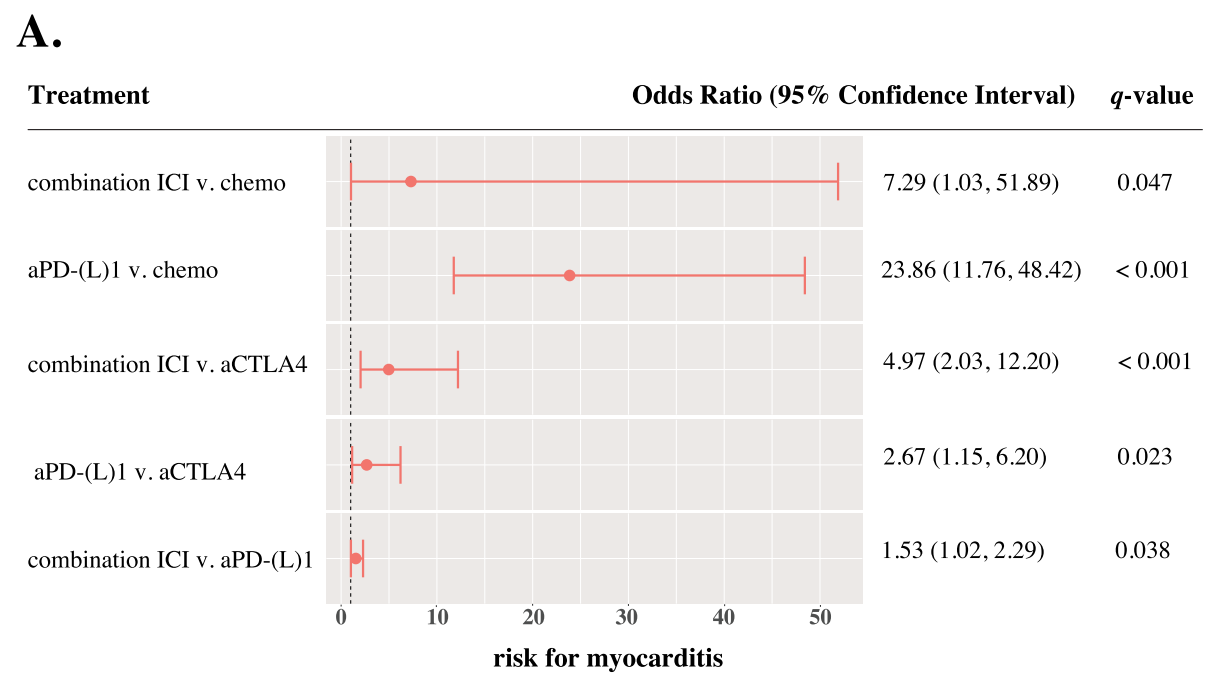

B.

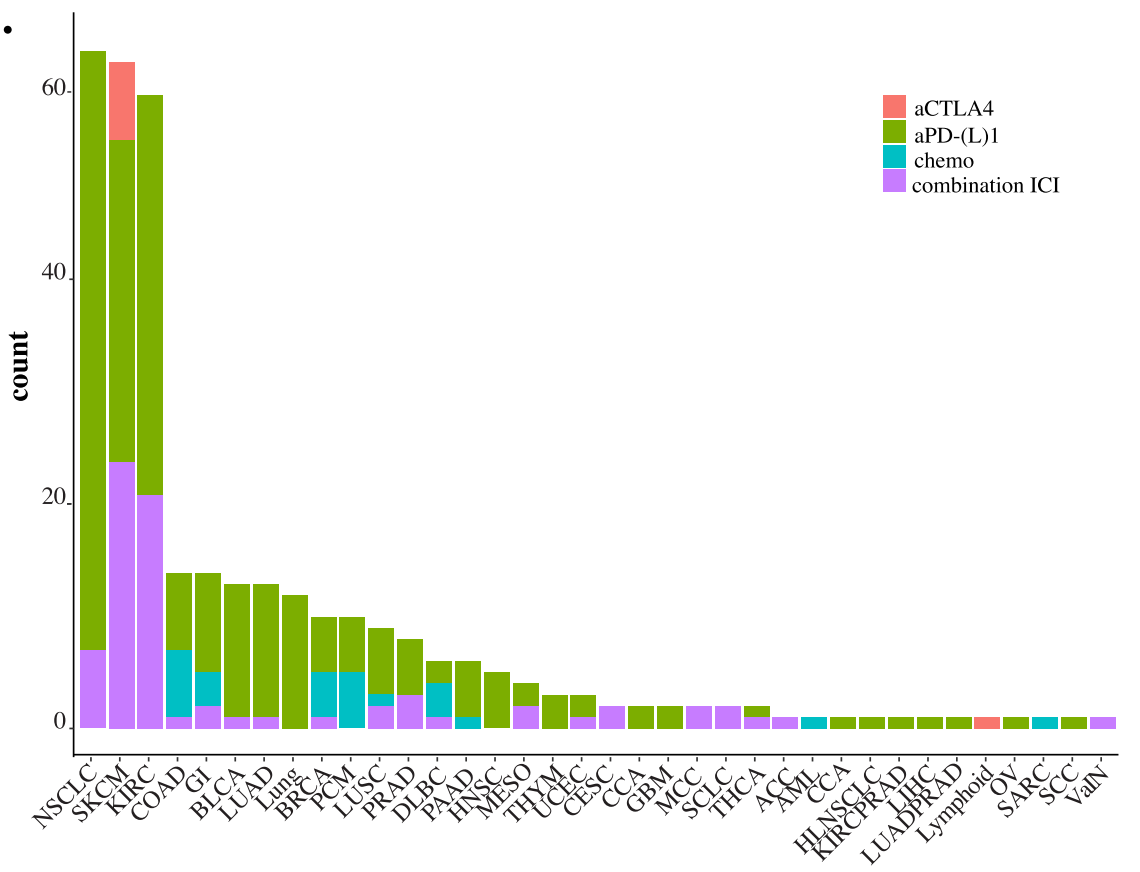

Fig. $1 \mathrm{Imm}$ unotherapy is significantly associated with myocarditis. a Forest plot represents matched logistic regression model results for myocarditis of cancer treatment groups (anti-PD-(L)1, anti-CTLA4, combination (more than one ICI), and chemotherapy). Shown are significant adjusted $p$-value $(q<.05)$ odds ratio and their 95\% confidence interval. Red circles represent an odds ratio greater than one, favoring immunotherapy. Blue circles indicate an odds ratio less than one, favoring chemotherapy. $\mathbf{b}$ Incidence of myocarditis by cancer histology is shown. Each color represents which treatment group the case corresponded to. Red corresponds to anti-CTLA4, green to anti-PD-(L)1, blue to chemotherapy, and purple to combination immunotherapy. Anti-CTLA4 = anti-cytotoxic T-lymphocyte-associated protein $4 ; \mid \mathrm{ICl}=\mathrm{immune}$ checkpoint inhibitor; anti-PD-(L)1 = anti-programmed death protein 1 and anti-programmed death-ligand 1

differences in the risk of cardiac adverse events in cancer patients.

\section{Discussion}

Our study provides comprehensive risk assessments of cardiac adverse events reports in cancer patients treated with immunotherapy and/or chemotherapy and includes 8300 cardiac adverse events reports. Although rare, cardiac adverse effects are shown to lead to serious consequences. Using the World Health Organization pharmacovigilance database, Wang et al. identified that cardiac and neurological events represent one-third of immune checkpoint inhibitor-related fatalities. The proportion of immunerelated fatalities were more frequent $(21 \%)$ in cases receiving more than one immunotherapy drug [11]. Among cardiac adverse events, others have found that ICI- 


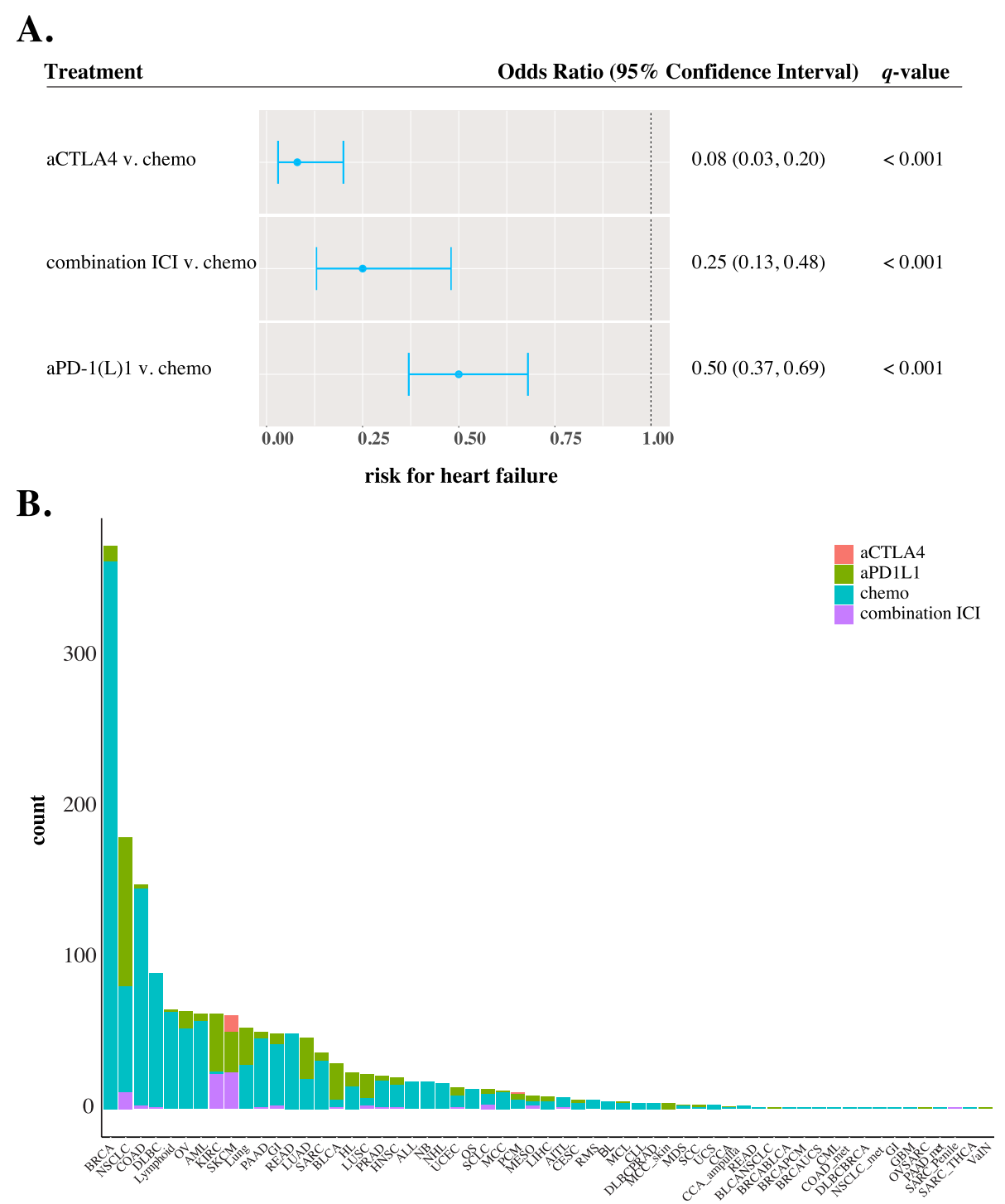

Fig. 2 Heart failure adverse events in cancer therapy. a Forest plot represents matched logistic regression model results for heart failure in cancer treatment groups (anti-PD- $(L) 1$, anti-CTLA4, combination (more than one ICI), and chemotherapy). Shown are significant adjusted $p$-value $(q<.05)$ odds ratio and their $95 \%$ confidence interval. Red boxes represent an odds ratio greater than one, favoring immunotherapy. Blue boxes indicate an odds ratio less than one, favoring chemotherapy. $\mathbf{b}$ Incidence of heart failure by cancer histology is shown. Each color represents which treatment group the case corresponded to. Red corresponds to anti-CTLA4, green to anti-PD-(L)1, blue to chemotherapy, and purple to combination immunotherapy. Anti-CTLA4 = anti-cytotoxic T-lymphocyte-associated protein 4; $\mid \mathrm{Cl}=$ immune checkpoint inhibitor; anti-PD-(L)1= anti-programmed death protein 1 and anti-programmed death-ligand 1

associated myocarditis has a mortality risk of $50 \%[11,18]$. Here, we validate that myocarditis remains disproportionally more common following ICI therapy compared to chemotherapy. This evidence reflects prior estimates of myocarditis using external databases $(<1 \%)[11,19,20]$. We also show that within this dataset myocarditis is more frequent in males. These findings follow previous reports of myocarditis occurring more frequently in men [11]. Furthermore, our analysis shows myocarditis reports were largely from patients with non-small cell lung cancer, melanoma, and renal cell carcinoma. This is likely a reflection of the cancer histologies receiving earlier FDA approval for immunotherapy. Despite higher surveillance of myocarditis leading to more reports in the last years, we find that myocarditis is disproportionally more common following immunotherapy (Fig. 1, Additional File 1: Supplemental Table 1).

The cause of interindividual variability in immunotherapy-mediated cardiotoxicity remains unclear. Clinical case reports have shown fatal cases of fulminant myocarditis following a single dose of anti-PD1 or antiCTLA4 treatment. Martinez-Calle et al. reported patients 


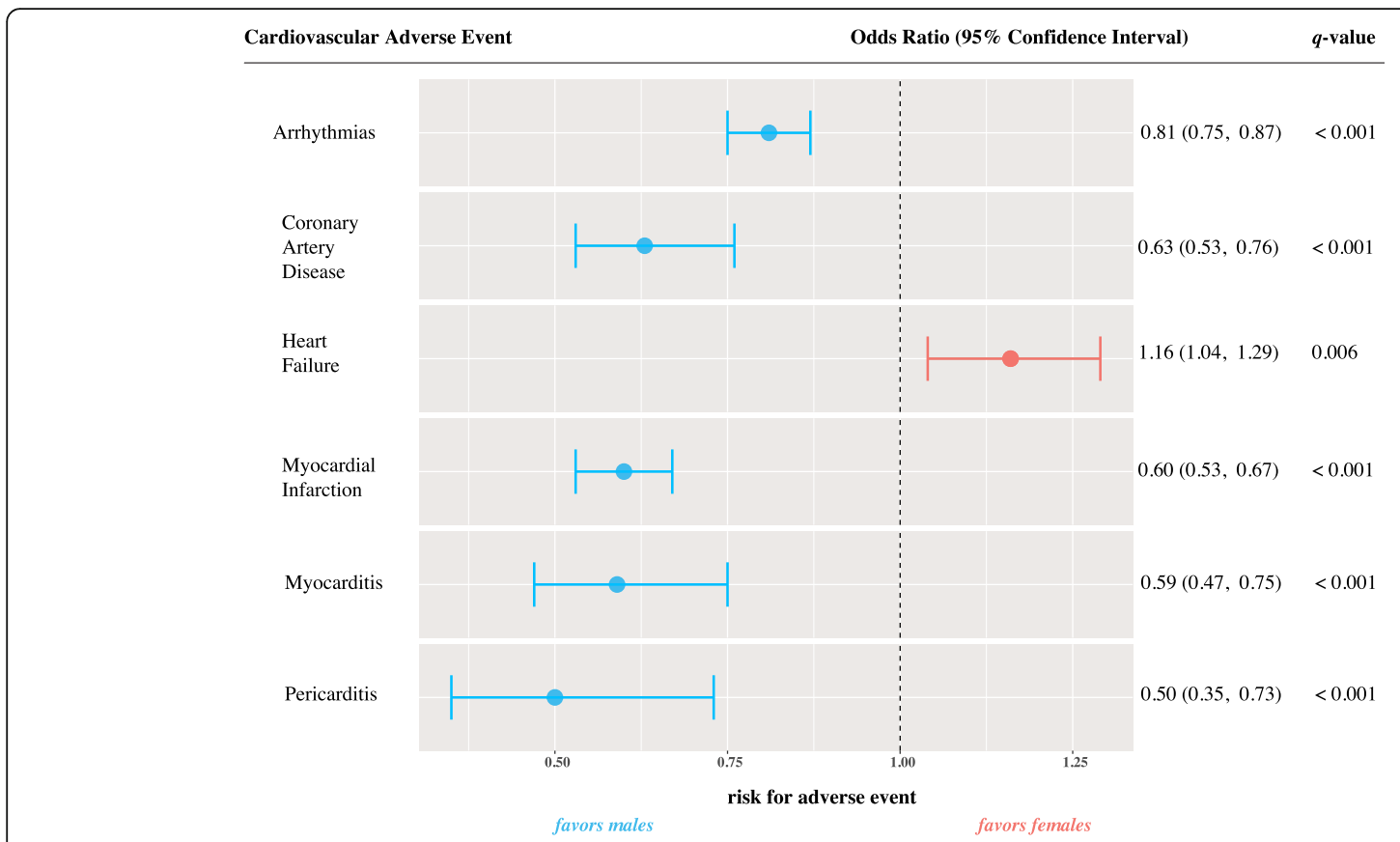

Fig. 3 Sex-specificity related to cardiac adverse events. Forest plots represent multiple logistic regression model results for cardiac adverse events, using males as a reference. Shown are significant adjusted $p$-values $(q<0.05)$ odds ratio and their $95 \%$ confidence interval. Red circles indicate an odds ratio greater than one, favoring females; blue circles indicate an odds ratio less than one, favoring males

tested positive for cardiac troponin auto-antibodies and had elevated troponin $\mathrm{T}$ levels suggesting a pre-existing $\mathrm{T}$ memory response that was abrogated by PD-1 blockade [21]. T cell receptor recognition in the heart thus results in a cytotoxic effect on cardiac tissue. Johnson et al. reported the presence of infiltrating lymphocytes and macrophages in the cardiac muscle. Lymphocyte receptor sequencing showed a significant overlap of TCR sequences among cardiac, skeletal, and tumor infiltrates, suggesting that the antigens in the myocardium and skeletal muscle were recognized by infiltrating lymphocytes [20]. However, we still do not fully understand which risk factors that may predispose a patient to lethal cardiac adverse events. Mechanistic studies to identify causality are imperative and underway.

Some opponents argue that the need for adding additional medical assessments for rare adverse events is an unnecessary burden for both patients and health care providers. Ederhy et al. highlighted clinical trials from 2010 to 2016 and reported only 15 cases of cardiac adverse events. A caveat to consider, however, is the lack of inclusion of patients with a medical history of cardiovascular disease and the lack of monitoring for cardiac related toxicity in ICI clinical trials [22]. Needless to say, pharmacovigilance reports have been critical in raising awareness and increasing surveillance. As a result, guidelines on clinical assessment, medical testing, intervention, and surveillance have been drafted to address this medical niche $[23,24]$. Since 2019, FAERS database reports of all cardiac adverse events have increased and now account for $50-60 \%$ of all cardiac adverse events in immunotherapy-related reports (Additional File 1: Supplemental Table 1). For this reason, we provide an updated analysis, to include a comparison with chemotherapy-related adverse events reports.

In this study, we also report a greater risk for heart failure in chemotherapy-treated patients. We observed that breast cancer represents the largest cancer histology among cases reported for heart failure. This finding likely reflects the well-established correlation of anthracycline-induced heart failure in breast cancer. Anthracycline-induced cardiotoxicity can occur as acute toxicity that results in arrhythmias or depressed left ventricular ejection fraction (LVEF), or chronic toxicity which results from excessive exposure or concomitant risk factors. The risk for developing heart failure increases with a high cumulative dose of doxorubicin [25]. However, only about half of patients show adverse effects at higher doses, indicating that heritable features might play a role in drug exposure, efficacy, and response [26]. A caveat to consider, reports of heart failure in breast cancer occur several years following drug exposure. With limited longitudinal data of immunotherapy since its initial approval in 2011, it may be too early to test whether heart failure is detected following ICI exposure [27].

We also report that immune-mediated cardiac adverse events are disproportionately more frequent in males. 
Table 2 Complete analysis of all cardiac AE risk from immunotherapy versus chemotherapy

\begin{tabular}{|c|c|c|c|}
\hline Adverse Event & Therapy & OR $(95 \% \mathrm{Cl})$ & q-value \\
\hline \multirow[t]{6}{*}{ Pericarditis } & Combination $v$ chemo & $0.61(0.23,1.67)$ & 0.339 \\
\hline & Combination $\vee$ aCTLA4 & $1.79(0.39,8.3)$ & 0.457 \\
\hline & Combination v aPD1L1 & $0.48(0.24,0.97)$ & 0.04 \\
\hline & aPD1L1 v chemo & $1.28(0.6,2.72)$ & 0.523 \\
\hline & aCTLA4 $\vee$ chemo & $0.34(0.07,1.66)$ & 0.184 \\
\hline & aCTLA4 $\vee$ aPD1L1 & $0.27(0.07,1.1)$ & 0.068 \\
\hline \multirow[t]{6}{*}{ Myocardial Infarction } & Combination $v$ chemo & $0.42(0.29,0.6)$ & $<0.001$ \\
\hline & Combination v aPD1L1 & $0.94(0.72,1.24)$ & 0.671 \\
\hline & Combination v aCTLA4 & $0.75(0.5,1.14)$ & 0.178 \\
\hline & aPD1L1 v chemo & $0.44(0.34,0.58)$ & $<0.001$ \\
\hline & aCTLA4 v chemo & $0.56(0.36,0.85)$ & 0.007 \\
\hline & aCTLA4 v aPD1L1 & $1.25(0.87,1.79)$ & 0.219 \\
\hline \multirow[t]{6}{*}{ Heart Failure } & Combination $v$ chemo & $0.38(0.27,0.54)$ & $<0.001$ \\
\hline & Combination $\vee$ aPD1L1 & $0.84(0.65,1.08)$ & 0.171 \\
\hline & Combination $v$ aCTLA4 & $2.21(1.22,3.9)$ & $<0.001$ \\
\hline & aPD1L1 v chemo & $0.46(0.36,0.59)$ & $<0.001$ \\
\hline & aCTLA4 $\vee$ chemo & $0.17(0.1,0.32)$ & $<0.001$ \\
\hline & aCTLA4 $\vee$ aPD1L1 & $0.38(0.22,0.66)$ & $<0.001$ \\
\hline \multirow[t]{6}{*}{ Coronary Artery Disease } & Combination $v$ chemo & $0.64(0.37,1.12)$ & 0.12 \\
\hline & Combination $v$ aCTLA4 & $1.47(0.59,3.63)$ & 0.405 \\
\hline & Combination v aPD1L1 & $0.96(0.6,1.52)$ & 0.848 \\
\hline & aPD1L1 v chemo & $0.67(0.47,0.96)$ & 0.028 \\
\hline & aCTLA4 $\vee$ chemo & $0.44(0.18,1.06)$ & 0.066 \\
\hline & aCTLA4 v aPD1L1 & $0.65(0.28,1.49)$ & 0.309 \\
\hline \multirow[t]{6}{*}{ Arrhythmias } & Combination v CTLA4 & $0.92(0.7,1.23)$ & 0.584 \\
\hline & Combination $v$ chemo & $0.71(0.61,0.84)$ & $<0.001$ \\
\hline & Combination v aPD1L1 & $1.06(0.89,1.26)$ & 0.531 \\
\hline & aPD1L1 v chemo & $0.67(0.61,0.73)$ & $<0.001$ \\
\hline & aCTLA4 $\vee$ chemo & $0.77(0.61,0.98)$ & 0.035 \\
\hline & aCTLA4 $\vee$ aPD1L1 & $1.15(0.89,1.47)$ & 0.289 \\
\hline \multirow[t]{6}{*}{ Myocarditis } & Combination v aPD1L1 & $1.41(1.08,1.85)$ & 0.012 \\
\hline & Combination $v$ chemo & $30.76(18.42,51.37)$ & $<0.001$ \\
\hline & Combination v aCTLA4 & $3.93(1.89,8.16)$ & $<0.001$ \\
\hline & aPD1L1 v chemo & $21.81(13.89,34.24)$ & $<0.001$ \\
\hline & aCTLA4 $\vee$ chemo & $7.83(3.41,18)$ & $<0.001$ \\
\hline & aCTLA4 v aPD1L1 & $0.36(0.18,0.73)$ & 0.005 \\
\hline
\end{tabular}

Shown is the logistic regression model data of the entire dataset, cases are not matched; Combination indicates more than one immunotherapy was administered. Adjusted p-values ( $q$-value) is displayed

aPD1L1 = anti- Programmed cell death protein - 1 and Programmed death ligand -1; aCTLA4 = cytotoxic T-lymphocyte- associated proteni 4

Several factors can lend to this observation. The occurrence of immune-related AEs is an indicator of ICI activity. Males are reported to have a better treatment effect of ICIs as reported in a meta-analysis from 20 randomized controlled trials [28]. More specifically, results from the KEYNOTE024 Phase 3 trial testing the efficacy of pembrolizumab
(anti-PD-1) in non-small cell lung cancer found that males exhibited a lower hazard for disease progression ( $\mathrm{HR}=$ $0.39,95 \%$ CI $0.26-0.58)$, compared to females $(\mathrm{HR}=0.75$, 95\% CI 0.46-1.21) [29]. Recent reports have investigated biological determinants of sex-specific responses to immunotherapy. Castro et al. have reported that sex 
differences relate to females exhibiting a strong immune selection early in tumorigenesis, which in turn leads to fewer driver mutations visible to the immune system at the time of ICI treatment [30]. This postulation corresponds well with recent reports from population GTEx data showing sex-biased expression of immunerelated pathways (antigen-presentation and T-cell proliferation) in females [31]. These compelling findings underscore the need to consider efficacy and risk assessment differently in males and females.

\section{Outlook}

Comprehensive identification of patients' specific predispositions, to individualize immunotherapy strategies and therefore yield the greatest clinical benefits at the lowest impact of heart and cardiovascular systems, is the ultimate goal of precision cardio-oncology and immunotherapy. Data mining of the large population registries, like the FAERS database, are important tools that allow for understanding clinical features correlated to drug adverse outcomes. However, such approaches will not be sufficient to identify causality. More comprehensive databases can further enrich our understanding of drug outcomes using pharmacogenomic approaches. The Clinical Pharmacogenetics Implementation Consortium, PharmGKB, and the RadioGenomics Consortium are examples of consortia that are focusing on evaluating genetic determinants of drug responses and adverse effects. Additionally, they help set guidelines for translating genotyping tests in cancer patients receiving treatment [32-34]. Individual national and hospital registries are also reporting major cardiovascular events using electronic health care data following cancer treatment $[8,35-38]$. These studies are important for validating $\mathrm{AE}$ incidence determined in pharmacovigilance studies and help identify additional important clinical determinants, such as the time of $\mathrm{AE}$ following drug exposure.

The next wave of health data availability will require advanced computational approaches for interpretation and medical applications. Artificial intelligence (AI) approaches to interpreting large-scale electronic health care record data, with machine learning algorithms will be critical to identify important features associated with cardiovascular adverse events [39]. To date, pioneering applications of artificial intelligence in the field of cardiology have focused on the interpretation of electrophysiology and cardiovascular imaging data [40-42]. Recently, Zhou et al. applied a durable machine learning model to assess six distinct cancer therapy-related cardiac events in 4309 cancer patients. The results identified 23 clinically relevant variables for at least one cardiac AE [39]. These research studies will be imperative to enable preventive measures of cardiac events in cancer patients. However, the precision of AI models' prediction and interpretation is limited by the quality of data, scale of the cohort, and availability and efficiency of computational resources [40].

\section{Study limitations}

We acknowledge several possible limitations in the current study, including heterogeneity and bias in selfreporting. We are not able to report risk estimates, given that our statistical analysis did not include cancer patients who did not experience adverse events following immunotherapy and, and thus we also cannot explain causality. We are not able to account for the probability of duplicate cases reported by health care professionals or the drug manufacturer and consumers. Although our statistical models adjusted for confounding factors, unmeasured confounding factors may exist as well. For example, the association of a drug with an adverse event might be explained by those of drugs that are coadministered. We also do not have information on cancer stage, or time of report following drug exposure, which could affect the associations. The database also lacks an indication of the medical history of cardiovascular disease prior to drug exposure. To overcome this limitation, we adjusted our model for the use of cardioprotective medications. Randomized controlled prospective studies will be imperative to validate findings, and ought to become clearer whether events are either a direct insult from cancer treatment or the exacerbation of underlying disease comorbidities (i.e., diabetes, hypertension, and existing cardiovascular conditions).

\section{Conclusion}

Here we report an elevated incidence of myocarditis in patients receiving immunotherapy compared to chemotherapy. Furthermore, we find that males are disproportionately at higher risk of immune-related cardiac adverse events. Additional clinical features can help expand the risk stratification of $\mathrm{AE}$ following ICI treatment. Understanding the clinical risk factors that predispose ICI-treated cancer patients to cardiotoxicity will be crucial in Cardio-Oncology management.

\section{Supplementary Information}

The online version contains supplementary material available at https://doi. org/10.1186/s40959-021-00106-x

\section{Additional file 1: Supplementary Figures.}

Additional file 2. Tumor Histology acronyms.

Additional file 3. Adverse Events keywords.

\section{Acknowledgements}

We thank all helpful discussions and critical comments regarding this manuscript from members at the Cleveland Clinic's Cardio-Oncology Center.

Authors' contributions

F.C. conceived the study. J.C.L. performed all experiments and data analysis. S.A.B. and P.C. helped to interpret the data analysis. J.C.L. and F.C. drafted the 
manuscript and critically revised the manuscript. All authors critically revised and gave final approval of the manuscript.

\section{Funding}

This work was supported by the National Heart, Lung, and Blood Institute of the National Institutes of Health (NIH) under Award Number K99 HL138272 and R00 HL138272 to F.C. This work has been also supported in part by the VeloSano Pilot Program (Cleveland Clinic Taussig Cancer Institute) to F.C.

\section{Availability of data and materials}

The datasets used and/or analysed during the current study are available from the FDA Adverse Event Reporting System (FAERS) Public Dashboard [12].

\section{Ethics approval and consent to participate} Not applicable.

\section{Consent for publication}

Not applicable.

\section{Competing interests}

The content of this publication does not necessarily reflect the views of the Cleveland Clinic. The authors declare no competing interests.

Dr. Sherry-Ann Brown is a member of the Cardio-Oncology Editorial Board.

\section{Author details}

${ }^{1}$ Genomic Medicine Institute, Lerner Research Institute, Cleveland Clinic Cleveland, $\mathrm{OH}$ 44195, USA. ${ }^{2}$ Department of Molecular Medicine, Cleveland Clinic Lerner College of Medicine, Case Western Reserve University, Cleveland, $\mathrm{OH}$ 44195, USA. ${ }^{3}$ Cardio-Oncology Program, Division of Cardiovascular Medicine, Medical College of Wisconsin, Milwaukee, WI 53226, USA. ${ }^{4}$ Robert and Suzanne Tomsich Department of Cardiovascular Medicine, Cleveland Clinic, Sydell and Arnold Miller Family Heart and Vascular Institute, Cleveland, $\mathrm{OH} 44195$, USA. ${ }^{5}$ Case Comprehensive Cancer Center, Case Western Reserve University School of Medicine, Cleveland, OH 44106, USA.

\section{Received: 20 January 2021 Accepted: 4 May 2021}

\section{Published online: 28 May 2021}

\section{References}

1. Schachter J, Ribas A, Long GV, Arance A, Grob JJ, Mortier L, et al. Pembrolizumab versus ipilimumab for advanced melanoma: final overall survival results of a multicentre, randomised, open-label phase 3 study (KEYNOTE-006). Lancet. 2017;390(10105):1853-62. https://doi.org/10.1016/ S0140-6736(17)31601-X.

2. Motzer RJ, Tannir NM, McDermott DF, Arén Frontera O, Melichar B, Choueiri TK, et al. Nivolumab plus Ipilimumab versus Sunitinib in advanced renal-cell carcinoma. N Engl J Med. 2018;378(14):1277-90. https://doi.org/10.1056/ NEJMoa1712126.

3. Rizvi NA, Mazières J, Planchard D, Stinchcombe TE, Dy GK, Antonia SJ, et al. Activity and safety of nivolumab, an anti-PD-1 immune checkpoint inhibitor for patients with advanced, refractory squamous non-small-cell lung cancer (CheckMate 063): a phase 2, single-arm trial. Lancet Oncol. 2015;16(3):25765. https://doi.org/10.1016/S1470-2045(15)70054-9.

4. Castrillon JA, Eng C, Cheng F. Pharmacogenomics for immunotherapy and immune-related cardiotoxicity. Hum Mol Genet. 2020;29(R2):R186-96. https://doi.org/10.1093/hmg/ddaa137.

5. Upadhaya S, Neftelino ST, Hodge JP, Oliva C, Campbell JR, Yu JX. Combinations take centre stage in PD1/PDL1 inhibitor clinical trials. Nat Rev Drug Discov. 2021;20(3):168-9.

6. Dougan M, Pietropaolo M. Time to dissect the autoimmune etiology of cancer antibody immunotherapy. J Clin Invest. 2020;130(1):51-61. https:// doi.org/10.1172/JCl131194.

7. Cautela J, Zeriouh S, Gaubert M, Bonello L, Laine M, Peyrol M, et al. Intensified immunosuppressive therapy in patients with immune checkpoint inhibitor-induced myocarditis. J ImmunoTher Cancer. 2020;8(2):e001887. https://doi.org/10.1136/jitc-2020-001887

8. D'Souza M, Nielsen D, Svane IM, Iversen K, Rasmussen PV, Madelaire C, et al. The risk of cardiac events in patients receiving immune checkpoint inhibitors: a nationwide Danish study. Eur Heart J. 2021;42(16):1621-31.
9. Bharadwaj A, Potts J, Mohamed MO, Parwani P, Swamy P, Lopez-Mattei JC, et al. Acute myocardial infarction treatments and outcomes in 6.5 million patients with a current or historical diagnosis of cancer in the USA. Eur Heart J. 2019;41(23):2183-93.

10. Wang DY, Salem JE, Cohen JV, Chandra S, Menzer C, Ye F, et al. Fatal toxic effects associated with immune checkpoint inhibitors: a systematic review and meta-analysis. JAMA Oncol. 2018;4(12):1721-8. https://doi.org/10.1001/ja maoncol.2018.3923.

11. Salem JE, Manouchehri A, Moey M, Lebrun-Vignes B, Bastarache L, Pariente A, et al. Cardiovascular toxicities associated with immune checkpoint inhibitors: an observational, retrospective, pharmacovigilance study. Lancet Oncol. 2018;19(12):1579-89. https://doi.org/10.1016/S1470-2045(18)30608-9.

12. FDA Adverse Events Reporting System Public Dashboard [https://www.fda gov/drugs/questions-and-answers-fdas-adverse-event-reporting-system-fa ers/fda-adverse-event-reporting-system-faers-public-dashboard) ].

13. Wickham H. stringr: Simple, Consistent Wrappers for Common String Operations; 2019.

14. Ho D, Imai K, King G, Stuart EA: Matchlt: Nonparametric Preprocessing for Parametric Causal Inference. 2011 2011, 42(8):28.

15. Mark Stevenson with contributions from Telmo Nunes $\mathrm{CH}$, Jonathon Marshall, Javier Sanchez, Ron Thornton, Jeno Reiczigel, Jim Robison-Cox, Paola Sebastiani, Peter Solymos, Kazuki Yoshida, Geoff Jones, Sarah Pirikahu, Simon Firestone, Ryan Kyle, Johann Popp, Mathew Jay and Charles Reynard epiR: Tools for the Analysis of Epidemiological Data. 2020.

16. Chongsuvivatwong V. epiDisplay: Epidemiological Data Display Package. In: vol. R package version 3.5.0.1; 2018.

17. Viechtbauer W: Conducting Meta-Analyses in $\mathrm{R}$ with the metafor Package. 2010 2010, 36(3):48.

18. Al-Kindi SG, Oliveira GH. Reporting of immune checkpoint inhibitorassociated myocarditis. Lancet. 2018:392(10145):382-3.

19. Hu JR, Florido R, Lipson EJ, Naidoo J, Ardehali R, Tocchetti CG, et al. Cardiovascular toxicities associated with immune checkpoint inhibitors. Cardiovasc Res. 2019;115(5):854-68. https://doi.org/10.1093/cvr/cvz026.

20. Johnson DB, Balko JM, Compton ML, Chalkias S, Gorham J, Xu Y, et al. Fulminant myocarditis with combination immune checkpoint blockade. N Engl J Med. 2016;375(18):1749-55. https://doi.org/10.1056/NEJMoa1609214.

21. Martinez-Calle N, Rodriguez-Otero P, Villar S, Mejías L, Melero I, Prosper F, et al. Anti-PD1 associated fulminant myocarditis after a single pembrolizumab dose: the role of occult pre-existing autoimmunity. Haematologica. 2018;103(7):e318-21. https://doi.org/10.3324/haematol.201 7.185777 .

22. Ederhy S, Voisin AL, Champiat S. Myocarditis with immune checkpoint blockade. N Engl J Med. 2017;376(3):290-1. https://doi.org/10.1056/NEJMc1 615251

23. Bonaca MP, Olenchock BA, Salem JE, Wiviott SD, Ederhy S, Cohen A, et al. Myocarditis in the setting of Cancer therapeutics: proposed case definitions for emerging clinical syndromes in cardio-oncology. Circulation. 2019;140(2): 80-91. https://doi.org/10.1161/CIRCULATIONAHA.118.034497.

24. Puzanov I, Diab A, Abdallah K, Bingham CO, Brogdon C, Dadu R, et al. Managing toxicities associated with immune checkpoint inhibitors: consensus recommendations from the Society for Immunotherapy of Cancer (SITC) toxicity management working group. J ImmunoTher Cancer 2017:5(1):95. https://doi.org/10.1186/s40425-017-0300-z.

25. Smith LA, Cornelius VR, Plummer CJ, Levitt G, Verrill M, Canney P, et al. Cardiotoxicity of anthracycline agents for the treatment of cancer: systematic review and meta-analysis of randomised controlled trials. BMC Cancer. 2010;10(1):337. https://doi.org/10.1186/1471-2407-10-337.

26. Allen A. The cardiotoxicity of chemotherapeutic drugs. Semin Oncol. 1992; 19(5):529-42.

27. Jensen BV, Skovsgaard T, Nielsen SL. Functional monitoring of anthracycline cardiotoxicity:a prospective, blinded, long-term observational study of outcome in 120 patients. Ann Oncol. 2002;13(5):699-709. https://doi.org/1 0.1093/annonc/mdf132.

28. Conforti F, Pala L, Bagnardi V, De Pas T, Martinetti M, Viale G, et al. Cancer immunotherapy efficacy and patients' sex: a systematic review and metaanalysis. Lancet Oncol. 2018;19(6):737-46. https://doi.org/10.1016/S1470-204 5(18)30261-4

29. Reck M, Rodríguez-Abreu D, Robinson AG, Hui R, Csőszi T, Fülöp A, et al. Pembrolizumab versus chemotherapy for PD-L1-positive non-small-cell lung Cancer. N Engl J Med. 2016:375(19):1823-33. https//doi.org/10.1056/ NEJMoa1606774. 
30. Castro A, Pyke RM, Zhang X, Thompson WK, Day C-P, Alexandrov LB, et al. Strength of immune selection in tumors varies with sex and age. Nat Commun. 2020;1 1(1):4128. https://doi.org/10.1038/s41467-020-17981-0

31. Oliva M, Muñoz-Aguirre M, Kim-Hellmuth S, Wucher V, Gewirtz ADH, Cotter DJ, et al. The impact of sex on gene expression across human tissues. Science. 2020;369(6509).

32. Barbarino JM, Whirl-Carrillo M, Altman RB, Klein TE. PharmGKB: A worldwide resource for pharmacogenomic information. Wiley Interdiscip Rev Syst Biol Med. 2018;10(4):e1417.

33. Kerns SL, Fachal L, Dorling L, Barnett GC, Baran A, Peterson DR, et al. Radiogenomics consortium genome-wide association study meta-analysis of late toxicity after prostate Cancer radiotherapy. J Natl Cancer Inst. 2020; 112(2):179-90. https://doi.org/10.1093/jnci/djz075.

34. Relling MV, Klein TE. CPIC: clinical Pharmacogenetics implementation consortium of the pharmacogenomics research network. Clin Pharmacol Ther. 2011;89(3):464-7. https://doi.org/10.1038/clpt.2010.279.

35. Hussain M, Hou Y, Watson C, Moudgil R, Shah C, Abraham J, et al. Temporal trends of cardiac outcomes and impact on survival in patients with Cancer. Am J Cardiol. 2020;137:118-24. https://doi.org/10.1016/j.amjcard.2020.09.030

36. Hussain M, Misbah R, Donnellan E, Alkharabsheh S, Hou Y, Cheng F, et al. Impact of timing of atrial fibrillation, CHA (2) DS (2)-VASc score and cancer therapeutics on mortality in oncology patients. Open Heart. 2020;7(2).

37. Lapirow D, La Gerche A, Toro C, Masango E, Costello B, Porello E, et al. The Australia and New Zealand Cardio-Oncology Registry: evaluation of chemotherapy-related cardiotoxicity in a national cohort of pediatric cancer patients. Intern Med J. 2021;51(2):229-34.

38. Mulrooney DA, Armstrong GT, Huang S, Ness KK, Ehrhardt MJ, Joshi VM, et al. Cardiac outcomes in adult survivors of childhood cancer exposed to cardiotoxic therapy. Ann Intern Med. 2016;164(2):93.

39. Zhou Y, Hou Y, Hussain M, Brown SA, Budd T, Tang WHW, et al. Machine learning-based risk assessment for Cancer therapy-related cardiac dysfunction in 4300 longitudinal oncology patients. J Am Heart Assoc. 2020; 9(23):e019628. https://doi.org/10.1161/JAHA.120.019628.

40. Xu B, Kocyigit D, Grimm R, Griffin BP, Cheng F. Applications of artificial intelligence in multimodality cardiovascular imaging: a state-of-the-art review. Prog Cardiovasc Dis. 2020;63(3):367-76. https://doi.org/10.1016/j.pca d.2020.03.003

41. Sevakula RK, Au-Yeung WTM, Singh JP, Heist EK, Isselbacher EM, Armoundas AA. State\&\#x2010;of\&\#x2010;the\&\#x2010;Art Machine Learning Techniques Aiming to Improve Patient Outcomes Pertaining to the Cardiovascular System. J Am Heart Assoc. 2020;9(4). https://doi. org/10.1161/JAHA.119.013924

42. Attia ZI, Noseworthy PA, Lopez-Jimenez F, Asirvatham SJ, Deshmukh AJ, Gersh BJ, et al. An artificial intelligence-enabled ECG algorithm for the identification of patients with atrial fibrillation during sinus rhythm: a retrospective analysis of outcome prediction. Lancet. 2019:394(10201):861-7. https://doi.org/10.1016/S0140-6736(19)31721-0.

\section{Publisher's Note}

Springer Nature remains neutral with regard to jurisdictional claims in published maps and institutional affiliations.

Ready to submit your research? Choose BMC and benefit from:

- fast, convenient online submission

- thorough peer review by experienced researchers in your field

- rapid publication on acceptance

- support for research data, including large and complex data types

- gold Open Access which fosters wider collaboration and increased citations

- maximum visibility for your research: over $100 \mathrm{M}$ website views per year

At $\mathrm{BMC}$, research is always in progress.

Learn more biomedcentral.com/submissions 